\title{
ERS/ECDC Statement: European Union standards for tuberculosis care, 2017 update
}

\author{
Giovanni Battista Migliori (10) ${ }^{1,26}$, Giovanni Sotgiu (102,26, Senia Rosales-Klintz ${ }^{3,26}$, \\ Rosella Centis (10) ${ }^{1,26}$, Lia D’Ambrosio (1) 1,4,26, Ibrahim Abubakar ${ }^{5}$, \\ Graham Bothamley ${ }^{6}$, Jose Antonio Caminero ${ }^{7,8}$, Daniela Maria Cirillo9 \\ Masoud Dara (10 ${ }^{10}$, Gerard de Vries ${ }^{11}$, Stefano Aliberti (10 ${ }^{12}$, Anh Tuan Dinh-Xuan ${ }^{13}$, \\ Raquel Duarte ${ }^{14}$, Fabio Midulla ${ }^{15}$, Ivan Solovic ${ }^{16}$, Dragan R. Subotic ${ }^{17}$, \\ Massimo Amicosante ${ }^{\dagger, 18}$, Ana Maria Correia ${ }^{19}$, Andra Cirule ${ }^{20}$, Gina Gualano ${ }^{21}$, \\ Heinke Kunst ${ }^{22}$, Fabrizio Palmieri ${ }^{21}$, Vija Riekstina ${ }^{23}$, Simon Tiberi ${ }^{22,24}$, \\ Remi Verduin ${ }^{25}$ and Marieke J. van der Werf (10),26
}

@ERSpublications

The European Union Standards for Tuberculosis Care: a guide for clinicians and public health workers to ensure optimal diagnosis, treatment and prevention of TB http://ow.ly/C80K30jxr1o

Cite this article as: Migliori GB, Sotgiu G, Rosales-Klintz S, et al. ERS/ECDC Statement: European Union standards for tuberculosis care, 2017 update. Eur Respir J 2018; 51: 1702678 [https://doi.org/10.1183/ 13993003.02678-2017].

ABSTRACT The International Standards for Tuberculosis Care define the essential level of care for managing patients who have or are presumed to have tuberculosis, or are at increased risk of developing the disease. The resources and capacity in the European Union (EU) and the European Economic Area permit higher standards of care to secure quality and timely TB diagnosis, prevention and treatment. On this basis, the European Union Standards for Tuberculosis Care (ESTC) were published in 2012 as standards specifically tailored to the EU setting. Since the publication of the ESTC, new scientific evidence has become available and, therefore, the standards were reviewed and updated.

A panel of international experts, led by a writing group from the European Respiratory Society (ERS) and the European Centre for Disease Prevention and Control (ECDC), updated the ESTC on the basis of new published evidence. The underlying principles of these patient-centred standards remain unchanged. The second edition of the ESTC includes 21 standards in the areas of diagnosis, treatment, HIV and comorbidities, and public health and prevention.

The ESTC target clinicians and public health workers, provide an easy-to-use resource and act as a guide through all the required activities to ensure optimal diagnosis, treatment and prevention of TB.

This document was endorsed by the by the ERS Science Council and Executive Committee in April 2018, and approved by ECDC in March 2018.

Received: Dec 222017 | Accepted after revision: March 112018

The content of this work is copyright of the authors or their employers. Design and branding are copyright @ERS 2018. 
Affiliations: ${ }^{1}$ World Health Organization Collaborating Centre for Tuberculosis and Lung Diseases, Maugeri Care and Research Institute, Tradate, Italy. ${ }^{2}$ Clinical Epidemiology and Medical Statistics Unit, Dept of Biomedical Sciences, University of Sassari, Sassari, Italy. ${ }^{3}$ European Centre for Disease Prevention and Control, Stockholm, Sweden. ${ }^{4}$ Public Health Consulting Group, Lugano, Switzerland. ${ }^{5}$ Institute for Global Health, University College London, London, UK. ${ }^{6}$ Homerton University Hospital, London, UK. ${ }^{7}$ Pneumology Dept, Hospital General de Gran Canaria “Dr. Negrin”, Las Palmas de Gran Canaria, Spain. ${ }^{8}$ MDR-TB Unit, Tuberculosis Division, International Union against Tuberculosis and Lung Disease (The Union), Paris, France. ${ }^{9}$ Emerging Bacterial Pathogens Unit, Div. of Immunology, Transplantation and Infectious Diseases, IRCCS San Raffaele Scientific Institute, Milano, Italy. ${ }^{10}$ World Health Organization, Regional Office for Europe, UN City, Copenhagen, Denmark. ${ }^{11}$ KNCV Tuberculosis Foundation, Den Haag, The Netherlands. ${ }^{12}$ School of Medicine and Surgery, University of Milan-Bicocca, UO Clinica Pneumologica, AO San Gerardo, Monza, Italy. ${ }^{13}$ Dept of Respiratory Physiology, Cochin Hospital, Paris Descartes University, Paris, France. ${ }^{14}$ National Reference Centre for MDR-TB, Hospital Centre Vila Nova de Gaia, Dept of Pneumology; Public Health Science and Medical Education Department, Faculty of Medicine, University of Porto, Porto, Portugal. ${ }^{15}$ Dept of Paediatrics, Paediatric Emergency Unit, “Sapienza" University of Rome, Rome, Italy. ${ }^{16}$ National Institute for TB, Lung Diseases and Thoracic Surgery, Vysne Hagy, Catholic University Ruzomberok, Ruzomberok, Slovakia. ${ }^{17} \mathrm{Clinic}$ of Thoracic Surgery, Belgrade, Serbia. ${ }^{18}$ Dept of Biomedicine and Prevention and Animal Technology Station, University of Rome "Tor Vergata", Rome, Italy. ${ }^{19}$ Regional Health Administration of the North, Dept of Public Health, Porto, Portugal. ${ }^{20}$ Centre of TB and Lung Diseases, Riga East University Hospital, Riga, Latvia. ${ }^{21}$ Respiratory Infectious Diseases Unit, National Institute for Infectious Diseases “L. Spallanzani”, IRCCS, Rome, Italy. ${ }^{22}$ Blizard Institute, Barts and The London School of Medicine and Dentistry, Queen Mary University, London UK. ${ }^{23}$ Dept of Methodology and Supervision, Riga East University Hospital, Riga, Latvia. ${ }^{24}$ Division of Infection, Royal London Hospital, Barts Health NHS Trust, London, UK. ${ }^{25}$ Verduin Public Health Consult, Oegstgeest, The Netherlands. ${ }^{26}$ Contributed equally.

Correspondence: G.B. Migliori, WHO Collaborating Centre for TB and Lung Diseases, Fondazione S. Maugeri, Care and Research Institute, via Roncaccio 16, 21049 Tradate, Italy.

E-mail: giovannibattista.miglioridicsmaugeri.it

Marieke J. van der Werf, European Centre for Disease Prevention and Control (ECDC), Gustav III:s boulevard 40, 16973 Solna, Sweden. E-mail: Marieke.vanderwerfdecdc.europa.eu

\section{Introduction}

With 58994 tuberculosis (TB) cases notified in the European Union and European Economic Area (EU/ EEA; refers to Austria, Belgium, Bulgaria, Croatia, Cyprus, Czech Republic, Denmark, Estonia, Finland, France, Germany, Greece, Hungary, Iceland, Republic of Ireland, Italy, Latvia, Liechtenstein, Lithuania, Luxembourg, Malta, The Netherlands, Norway, Poland, Portugal, Romania, Slovakia, Slovenia, Spain, Sweden and the UK) member states in 2016 [1], TB continues to be a priority public health challenge in this setting. Although several EU/EEA countries are progressing towards sustained low levels of TB incidence and some towards the pre-elimination phase (defined as less than 10 cases per million population [1-3]), great diversity in TB disease burden remains within the sub-region [1]. Drug-resistant TB, multidrug-resistant TB (MDR-TB) and extensively drug-resistant TB (XDR-TB) pose a specific public health threat in many countries [4]. Furthermore, documenting the prevalence of HIV co-infection among TB cases is still compromised by sub-optimal reporting in several countries $[5,6]$.

While EU/EEA countries adopted the key principles of TB control and elimination through the Europe-specific, consensus-based documents born within the Wolfheze initiative [7] and subsequent documents [2], a uniform set of guidelines summarising essential standards to guide European clinicians and healthcare workers was developed only in 2012 [8]. These European Union Standards for Tuberculosis Care (ESTC) were based on the second version of the International Standards for Tuberculosis Care (ISTC) issued in 2009 [9]. The documents were developed by experts selected to include the perspectives of several countries, organisations, national TB programmes, and scientific and civil societies, as well as representatives of the affected communities, which endorsed them for universal (ISTC) or EU/EAA countries' use (ESTC).

Both ISTC and ESTC [8-11] prescribe a widely accepted level of TB care, to guide all healthcare providers and clinicians, both public and private, in achieving optimal standards in managing individuals who have active TB, latent TB infection (LTBI) or signs and symptoms compatible with the disease. The standards are designed to complement existing national or international guidelines and are consistent with World Health Organization (WHO) definitions and recommendations [12].

The original ESTC include 21 standards organised into four sections: 1) standards for diagnosis, 2) standards for treatment, 3) standards for addressing HIV co-infection and other comorbidities and 4) standards for public health [8]. The standards are a living document, and will thus be revised as technology and circumstances change. In 2014, the third edition of the ISTC was published [10]. An assessment and a survey among European Respiratory Society (ERS) members and European Centre for Disease Prevention and Control (ECDC) contacts concluded that a revision of the ESTC would be appropriate $[13,14]$. 
The specific mix of characteristics among the EU/EEA member states which justified the development of standards specifically tailored to the EU context are still valid, and are summarised below $[8,11,15]$ :

1) Although the majority of EU/EEA countries have a low incidence of TB, a number of member states have intermediate TB levels, with varying incidence of MDR-TB and TB-HIV co-infection. Furthermore, several countries located at the eastern border of the EU are neighbours to non-EU countries with a high TB and MDR-TB burden.

2) TB services are fully integrated and merged within the health system in the majority of EU/EEA countries. This leads to individual country peculiarities in allocating responsibilities for the optimal delivery of TB care.

3) The EU/EEA countries have a long-established tradition of TB control that has evolved over past decades. New tools and high standards of diagnosis and care are often implemented in EU/EEA countries.

4) The EU/EEA is committed to pursuing the elimination of TB, sharing a common platform based on the Wolfheze documents and subsequent documents; the Global End TB Strategy and its related action plan specific to the European region; as well as the surveillance system driven by ECDC and WHO Regional Office for Europe [2, 16-22].

The purpose of this document is to incorporate the new scientific evidence that has become available after the publication of the ESTC in 2012.

\section{Methods}

A collaborative process, jointly led by the ERS and the ECDC, was initiated in 2016 to revise the ESTC. The process received input from an ERS task force. The task force included a panel of experts representing the ERS, other international societies and organisations (World Health Organization, the UNION, KNCV Tuberculosis Foundation), national TB programmes, civil society, and affected communities. All task force members provided a conflict of interest declaration, in line with ERS regulations.

This update builds upon the main areas outlined in the previous ESTC edition, i.e. diagnosis, treatment, HIV and comorbidities, and public health and prevention. The ERS has taken the lead in developing the clinically related standards and ECDC has done the same for the public health standards. After conducting an initial scoping search, it was determined that sufficient relevant evidence was already available for an update of ESTC. Hence, no systematic reviews were conducted as part of the ESTC updating process.

New evidence was identified through a targeted, non-systematic literature search. The search focused on evidence-based guidelines, systematic reviews/meta-analyses, peer-reviewed scientific articles, institutional reports and policy recommendations produced after the publication of the first edition of the ESTC (i.e. 2012). Relevant evidence was retrieved after consulting the expert panel, institutional websites and selected electronic databases, i.e. Medline (www.ncbi.nlm.nih.gov/pubmed/), PROSPERO (www.crd.york.ac.uk/ PROSPERO/) and the Cochrane Database of Systematic Reviews (www.cochranelibrary.com/).

Task force members assessed the synopsis of the evidence and provided their written input for the revision of the 21 standards and their supporting enablers for implementation. A writing committee consisting of six experts (four from ERS and two from ECDC) led the writing process of the document (identified as "equal contributors" in the author list). After three discussion rounds, consensus was reached and the final document was approved. All co-authors participated in the entire process and contributed to the final document.

This document provides up-to-date standards for tuberculosis care tailored to the EU and the EEA on the basis of recently published policy statements and international guidelines, in particular those listed in table 1.

The final ESTC manuscript was developed according to the ERS rules for official statements before being peer reviewed by the European Respiratory Journal. In parallel the document gained clearance by ECDC.

\section{How to read the document}

Divided into four sections (diagnosis, treatment, HIV and comorbidities, and public health), 21 standards are defined, following the structure of the first version of the ESTC. For each standard, it is indicated if, in comparison with the previous version of the ESTC, a given standard remained unchanged or underwent modifications. Table 2 summarises all the changes. Under the heading "EU-specific requirements" further considerations are provided. When necessary, notes are listed after each standard, to further define and explain specific components that the expert group deemed important to clarify. 
TABLE 1 Core documents consulted for this update

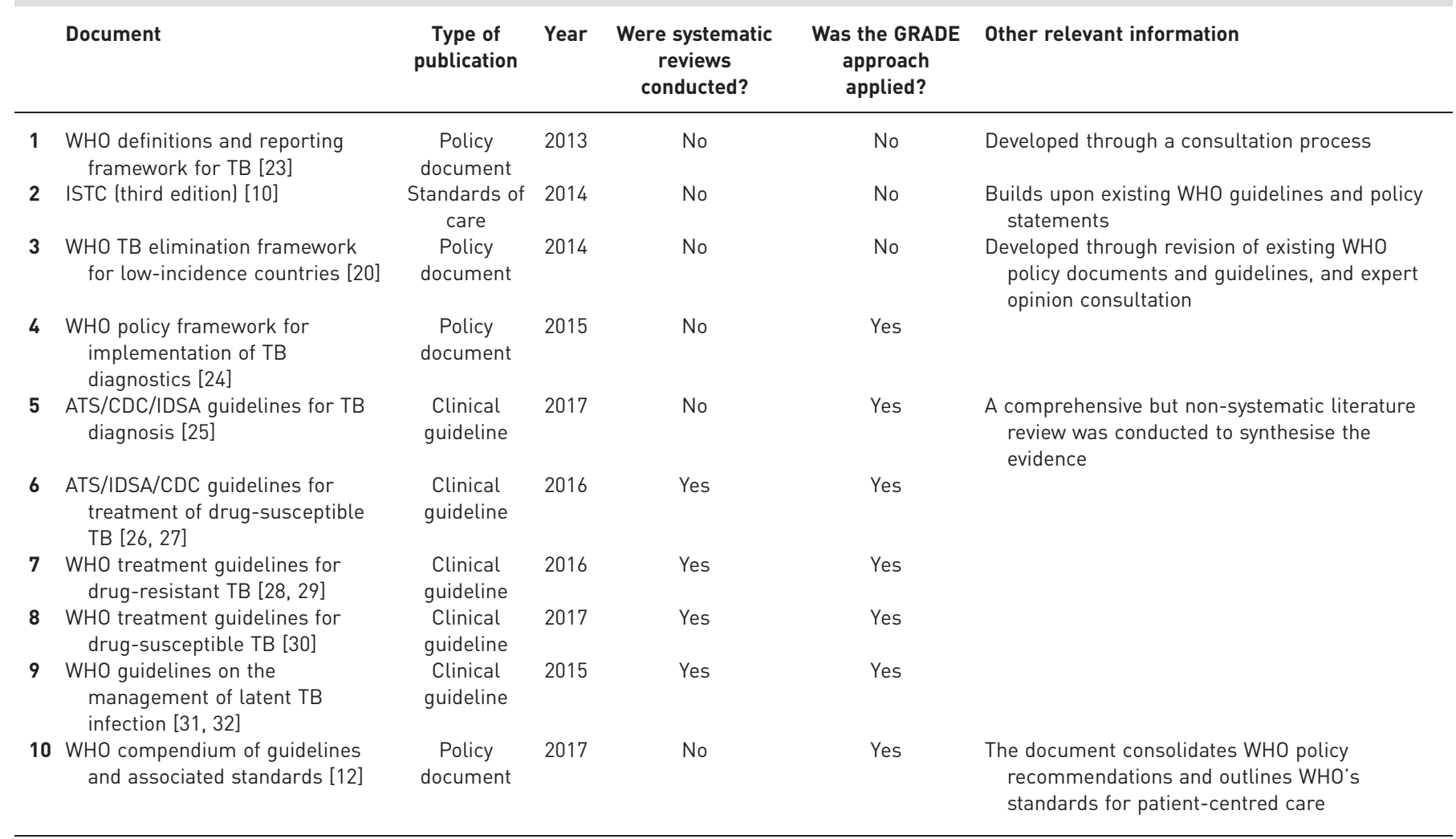

GRADE: Grading of Recommendations Assessment, Development and Evaluation; WHO: World Health Organization; TB: tuberculosis; ISTC: International Standards for Tuberculosis Care; ATS: American Thoracic Society; CDC: Centers for Disease Control and Prevention; IDSA: Infectious Diseases Society of America.

\section{Standards for tuberculosis diagnosis Standard 1 (unchanged)}

All persons presenting with signs, symptoms, history or risk factors compatible with tuberculosis should be evaluated for pulmonary and/or extrapulmonary tuberculosis.

Notes

The most common symptom of pulmonary TB is persistent cough with or without sputum production for more than 2-3 weeks [10], while haemoptysis (coughing up blood) is more unusual. These signs and symptoms are common in a wide range of respiratory conditions including acute respiratory infections (ARI) and acute exacerbation of chronic obstructive pulmonary disease (COPD). Respiratory symptoms can be accompanied by fever, night sweats and weight loss. For extrapulmonary TB, organ-specific signs and symptoms may occur. Individuals may also have TB, without specific signs and symptoms of disease, especially those who are immunosuppressed [33].

It is important to question the patient with regard to TB. For example, a history of TB in the family, history of previous contact with TB as well as previous TB diagnosis and/or treatment, and any condition attenuating the host immune system [10] are common risk factors for TB that should be considered as relevant to the diagnosis. TB in a child is always a "sentinel event" for recent transmission (e.g. a source case is likely to exist in the family or in their class [34]).

In the EU setting, TB is not the leading cause of persistent cough. Also, cough is not necessarily the most common symptom of TB disease [35].

Standard 2 (changed)

All patients (adults, adolescents and children who are capable of producing sputum) thought to have pulmonary tuberculosis should have at least two sputum specimens submitted for microscopic examination and one for rapid testing for the identification of tuberculosis and drug resistance using an internationally recommended (rapid) molecular test. The sample should be sent for liquid culture and, if 
TABLE 2 Summary of changes in the second edition compared to the first edition of the European Union Standards for Tuberculosis Care (ESTC)

\section{Standard}

\section{Comparison with ESTC Description of the update}

(first edition)

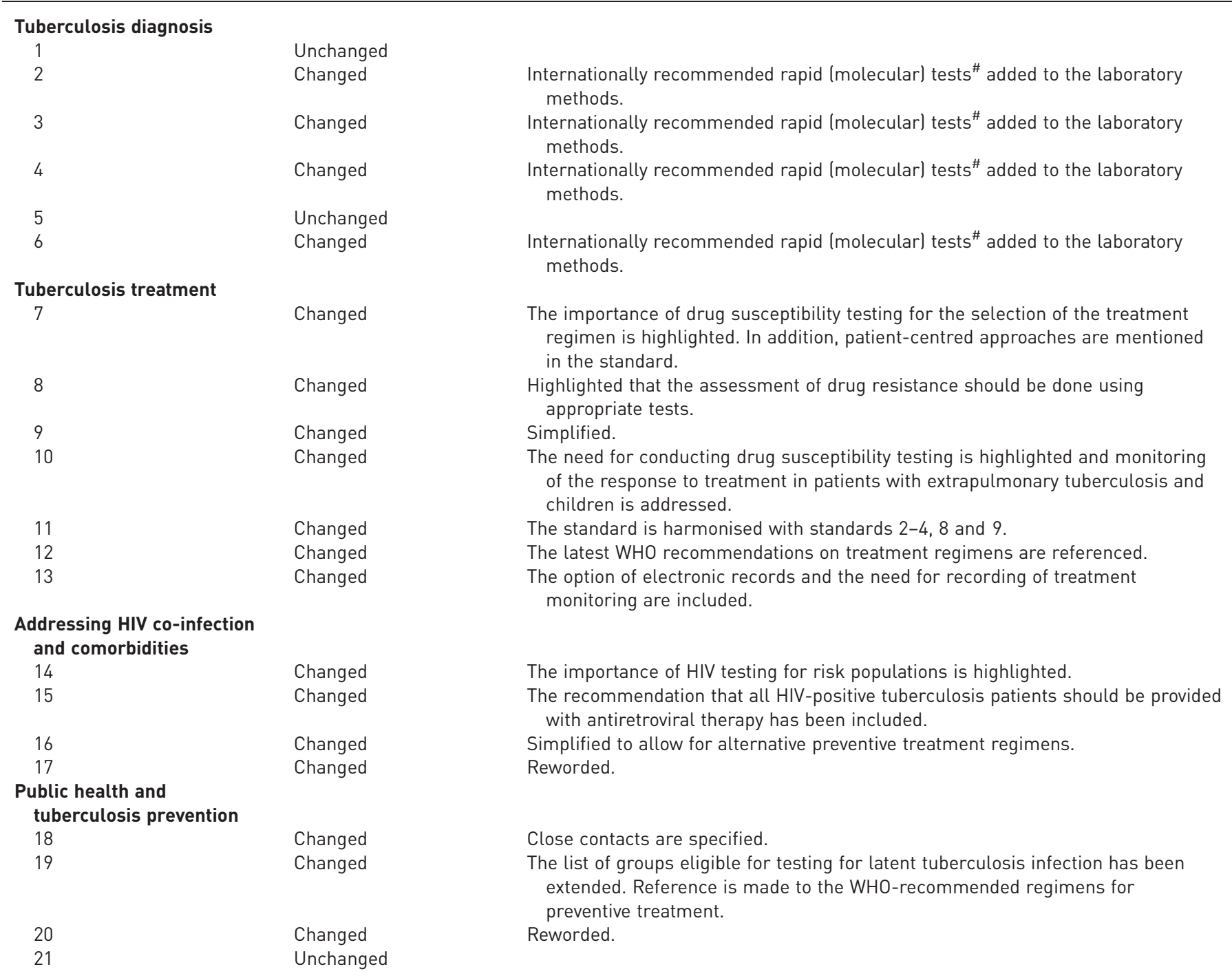

WHO: World Health Organization. " : the term "internationally recommended rapid (molecular) tests" includes WHO-recommended tests.

positive, for culture-based drug susceptibility testing (DST) in a quality-assured laboratory. When possible, at least one early morning specimen should be obtained. Chest radiography can also be used.

Notes

The order of execution of the microbiological tests mentioned in this and in the following standards will depend on the laboratory workflow.

The term "internationally recommended (rapid) molecular tests" refers to diagnostic tests validated by internationally recognised organisations, including WHO. From this point onwards these tests will be referred to as "rapid molecular tests".

Based on the EU/EEA practice and definitions [36, 37], quality-assured DST should be performed on all diagnosed TB patients to rule out drug resistance [16, 37-40]. This should follow international standards and guidelines with regard to methods used and drug concentrations, for testing of first- and second-line drugs $[16,18]$. Samples sent for bacteriological examinations (sputum smear, culture, DST, new molecular methods) should be addressed to a mycobacteriology laboratory which implements 
optimal laboratory practices and quality assured procedures according to European and international recommendations $[16,18,25]$.

Quality bacteriological diagnosis includes the WHO-recommended rapid (molecular) assays [12, 41-46]; it should be done as early as possible (ideally initiated on the day the presumed patient is identified) within evidence-based diagnostic algorithms and guidelines. Currently available genotypic methods are the automated real-time nucleic acid amplification technology for rapid and simultaneous detection of Mycobacterium tuberculosis and rifampicin resistance (e.g. the GeneXpert platform) and the line probe assays for rapid $M$. tuberculosis detection and rifampicin resistance or rifampicin- and/or isoniazid-resistance testing $[12,46-48]$. These approaches allow immediate identification of $M$. tuberculosis and rifampicin resistance and/or MDR-TB (rifampicin resistance can be considered a proxy of MDR-TB) $[12,41-46,49,50]$. Whole genome sequencing (WGS) and WGS-based tools are available for diagnosis and identification of $\mathrm{TB}$ and of drug resistant variants; they are used in some EU countries [51-53]. Molecular diagnostic results must be confirmed by phenotypic testing, i.e. culture-based DST [38, 49, 50]; in case of discrepant results sequencing could be performed from the isolate to identify the mutation and, in case of high confidence mutations, the isolate should be regarded as resistant. The culture-based DST confirmation should be done by quality-assured laboratories [54, 55].

At present the choice of molecular methods for the rapid identification of rifampicin and isoniazid resistance should be that of WHO-recommended rapid diagnostic assays [12, 29]. We expect that in the near future the role of sequencing as a reference for molecular tests will be recognised and additional molecular tests will become available in Europe.

Timely, clear and direct communication between the laboratory experts and clinicians is essential to obtain the optimal link between diagnosis and treatment regimen. The clinician needs to be informed whether the laboratory performs DST for second-line drugs, and on which drugs [16].

Given that the collection of a third sputum sample has been shown to increase the diagnostic yield by $2-3 \%$, EU/EEA-countries may decide to maintain the previous recommendation of collecting three sputum samples on the same day (not necessarily on consecutive days) [10, 56].

It is essential to obtain good quality sputum samples in order to ensure reliable bacteriological testing of the sample $[10,56]$. This includes providing clear instructions to the patient, and ensuring appropriate collection, storage, transportation and processing of sputum samples [57].

To ensure rapid diagnosis a sputum sample should be collected as soon as possible. In addition, it is recommended to obtain at least one early morning sample from the patient.

Every effort should be made to obtain samples for culture and DST, using the different procedures available according to evidence-based guidelines (sputum induction, bronchoscopy/bronchoalveolar lavage and gastric lavage in children $[58,59])$.

All MDR-TB strains should be collected and stored at the national level for monitoring drug-resistance trends and map national and cross-border clusters.

\section{Standard 3 (changed)}

For all patients (adults, adolescents and children) presumed to have extrapulmonary tuberculosis, appropriate specimens from the suspected sites of involvement should be obtained for microbiological testing (microscopy, rapid molecular tests, culture, species identification, DST with rapid molecular tests and culture-based techniques) and histopathological examination in quality-assured laboratories.

Notes

This third standard has been accordingly updated to be in line with standard 1 and 2 with regard to essential, standard diagnosis. So, rapid molecular testing is recommended for all cases [12].

It is essential to use all efforts to obtain bacteriological confirmation from extrapulmonary sites in order to confirm diagnosis, allow DST and consequently provide optimal and effective treatment; this may include a more sensitive molecular test [10].

\section{Standard 4 (changed)}

All persons with chest radiographic findings suggestive of pulmonary tuberculosis should have sputum specimens submitted for microscopic examination, rapid molecular tests, culture, species identification and DST with rapid molecular tests and culture-based techniques in a quality-assured laboratory. 
Note

This standard has been updated to be consistent with the standards 1 and 2 with regard to essential, standard diagnosis, including rapid molecular testing $[10,12]$.

\section{Standard 5 (unchanged)}

The diagnosis of culture-negative pulmonary tuberculosis should be based on the following criteria: all bacteriological tests are negative (including direct sputum smear examinations, cultures and rapid molecular testing); chest radiographic findings compatible with tuberculosis; and lack of response to a trial of broad spectrum antimicrobial agents (note: because the fluoroquinolones are active against M. tuberculosis complex, and may cause transient improvement in persons with tuberculosis, their use should be avoided). In persons who are seriously ill or have known or presumed HIV infection or have any immune-compromising conditions, the diagnostic evaluation should be expedited and, if clinical evidence strongly suggests tuberculosis, a course of anti-tuberculosis treatment should be initiated.

\section{EU-specific requirements}

In order to ensure quality diagnosis of both pulmonary and extrapulmonary tuberculosis, adequate samples for bacteriologic examination should be obtained. Sputum induction, bronchoscopy and bronchoalveolar lavage, gastric washing, biopsy or fine needle aspiration should be used where appropriate [60]. Samples should be processed using available diagnostic tools [10], and complemented by imaging (radiology, ultrasound, computed tomography, magnetic resonance imaging, positron emission tomography-computed tomography) and other necessary examinations performed according to evidence-based guidelines $[10,25,61]$.

WHO-recommended rapid molecular testing [12], culture and DST should be performed on each sample from patients with presumed pulmonary and extrapulmonary $\mathrm{TB}$, including samples obtained during surgery or other invasive procedures which usually undergo histological examinations. Surgeons should thus be advised to save a biological specimen in normal saline for microbiological and molecular biological examinations and in formalin for histopathological examinations.

Note

Other existing new diagnostic tools, e.g. additional molecular tests and other new techniques in the development pipeline, should be used within evidence-based diagnostic algorithms and guidelines. Before introducing any new tool or approach, the evidence has to be validated and have shown efficacy and patient value.

\section{Standard 6 (changed)}

In all children presumed to have intrathoracic (i.e., pulmonary, pleural, and mediastinal or hilar lymph node) tuberculosis, bacteriological confirmation should be sought through examination of appropriate biological samples (by expectorated or induced sputum, bronchial secretions, pleural fluid, gastric washings or endoscopic ultrasound-guided biopsy) by smear microscopy, rapid molecular tests, species identification and DST with culture-based techniques in a quality-assured laboratory [12, 16-18, 25, 62]. In the event of negative bacteriological results, a diagnosis of tuberculosis should be based on the presence of abnormalities consistent with tuberculosis on chest radiography or other imaging, a history of exposure to an infectious case, evidence of tuberculosis infection (positive tuberculin skin test (TST) and/or a positive interferon-gamma release assay (IGRA)) [12, 63-66], and/or clinical findings suggestive of tuberculosis [25]. For children presumed to have extrapulmonary tuberculosis, appropriate specimens from the suspected sites of involvement should be obtained for microscopy, recommended rapid molecular tests, species identification and DST with culture-based techniques; and histopathological examination $[12,59,67]$.

\section{Standards for tuberculosis treatment Standard 7 (changed)}

Any practitioner treating a patient for tuberculosis is assuming an important public health responsibility to prevent ongoing transmission of the infection and the development of drug resistance. To fulfil this responsibility, the practitioner, in collaboration with public health authorities, must: 1) prescribe an appropriate regimen (guided by the genotypic and/or phenotypic DST results); 2) perform contact investigations; 3) assess and promote the patient's adherence to treatment using a patient-centred approach in collaboration with family members, local public and/or community health services, and civil society organisations; and 4) monitor treatment outcomes [10, 68, 69]. 


\section{Standard 8 (changed)}

All patients (including those with HIV co-infection) who have not been previously treated and are without drug resistance (assessed by appropriate tests) should receive an internationally accepted first-line treatment regimen using drugs of known bioavailability. The initial phase should consist of 2 months of isoniazid $(\mathrm{H})$, rifampicin $(\mathrm{R})$, pyrazinamide $(\mathrm{Z})$ and ethambutol $(\mathrm{E})$. The continuation phase should consist of isoniazid and rifampicin given for 4 months (2HRZE/4HR). The doses of anti-tuberculosis drugs used should conform to international recommendations. Fixed dose combinations of two (isoniazid and rifampicin), three (isoniazid, rifampicin, and pyrazinamide) and four (isoniazid, rifampicin, pyrazinamide, and ethambutol) drugs may provide a more convenient form of drug administration.

\section{Notes}

The clinician should ensure the correct drug regimen (including four drugs for the intensive phase of treatment) at the correct dose for a sufficient duration. Daily dosage is strongly recommended [10, 12, 26, $28,55]$. The continuation phase of treatment can be initiated if susceptibility to isoniazid and rifampicin is confirmed.

It is suggested to treat cases with rifampicin mono-resistance and MDR-TB in centres with experience, to allow close patient monitoring and adaptation of the treatment regimen on the basis of latest scientific evidence and forthcoming updated recommendations.

Rifampicin blood levels may be monitored if poor response to treatment due to under-dosing or malabsorption is suspected $[26,27]$.

The 2017 WHO TB treatment guidelines advocated the use of adjuvant corticosteroid therapy with dexamethasone or prednisone during the first 6-8 weeks for $\mathrm{TB}$ meningitis [30], in $\mathrm{TB}$ pericarditis to prevent constrictive pericarditis and avoid surgery, in renal TB to prevent ureteric stenosis and in spinal TB (if evidence of spinal cord compression) [30].

\section{Standard 9 (changed)}

A patient-centred approach to treatment, based on the patient's needs and mutual respect between the patient and the provider, should be developed for all patients.

\section{Notes}

A central element of the patient-centred strategy is the use of measures to assess and promote adherence to the treatment regimen and to address poor adherence. These measures should be tailored to the individual patient's circumstances, based on a detailed clinical and social history, and be mutually acceptable to the patient and the provider.

Supervision and support should be individualised and should draw on the full range of recommended interventions and available support services. The aim is to prevent poor adherence before it occurs. Such measures may include direct observation of medication ingestion (directly observed treatment (DOT) or video-observed treatment (VOT)) and/or identification and training of a treatment supporter (for tuberculosis and, if appropriate, for HIV infection) who is acceptable and accountable to the patient and to the health system. Appropriate incentives and enablers, including financial, social and psycho-social support, may also serve to enhance treatment adherence [10, 12, 26, 27, 30, 70, 71].

Additional services included in the package of interventions recommended in the 2017 WHO TB treatment guidelines to support the patient (with drug-susceptible or drug-resistant TB) and promote adherence $[12,26,27,30,70,71]$ are: 1) health education and counselling; 2) tracers or digital medication monitoring methods; 3) material support to the patients; 4) staff education; and 5) community or home-based observed treatment.

A decentralised model of service delivery for MDR-TB care is recommended. This approach is more convenient for patients and might ensure economic savings in some settings.

\section{Standard 10 (changed)}

Response to therapy in patients with pulmonary tuberculosis should be monitored by follow-up smear microscopy and culture, at least, at the time of completion of the initial phase of treatment (two months for drug-susceptible tuberculosis). If the sputum smear and/or culture are positive at completion of the initial phase, molecular tests of drug resistance and further DST should be performed promptly. In patients with extrapulmonary tuberculosis and in children unable to produce sputum, the clinical response to treatment (weight, inflammatory markers and repeat imaging) is objectively assessed. 


\section{EU specific requirements}

Treatment monitoring should be done according to international guidelines [10, 16-18, 23, 25-27]. In the EU, countries have resources to perform treatment monitoring on a monthly basis. For MDR-TB cases, this monthly monitoring should be done based on sputum smear and culture [54, 72].

\section{Standard 11 (changed)}

An assessment of the likelihood of drug resistance, based on history of prior treatment, exposure to a possible source case with drug-resistant $\mathrm{TB}$, and the community prevalence of drug resistance, should be made, especially for patients who are not bacteriologically confirmed or for whom drug susceptibility testing cannot be performed. Rapid testing (genotypic rifampicin and isoniazid resistance testing and genotypic/phenotypic second-line drug resistance testing for patients with rifampicin resistance or MDR-TB) should be performed for all patients as defined in standards 2-4 and 8. Furthermore, patient counselling and education should begin immediately for all tuberculosis patients, in order to minimise the potential for transmission. Infection control measures appropriate to the setting should be applied as recommended in ESTC public health standard 20.

\section{Notes}

This standard emphasises the need to use rapid molecular tests to rule-out or confirm presumed MDR-TB as described in standards 2-4 and 8 [12]. In the near future, other genotypic techniques such as WGS could be considered.

As expressed in standards 2-4, rapid molecular testing for rifampicin and isoniazid resistance does not yet rule out the requirement to perform standard DST to confirm results from the molecular test as well as perform the comprehensive standard DST for other drugs.

Resistance to other second line drugs in Europe is common [73-78].

Phenotypic DST should only be performed and interpreted for drugs with critical concentration and clinical breakpoints established and fully validated [12, 53].

Genotypic DST should be interpreted based on an agreed list of mutations and interpretations [12, 53]

\section{Standard 12 (changed)}

Patients with, or highly likely to have, tuberculosis caused by drug-resistant (especially rifampicin-resistant/MDR/XDR) organisms should be treated with individualised regimens containing second-line and add-on anti-tuberculosis drugs. The regimen chosen should be based on confirmed drug susceptibility patterns. Empirical regimens may cause further resistance and are not recommended, except for culture-negative tuberculosis.

Depending on the drug susceptibility pattern, treatment with a minimum of five effective anti-TB drugs should be provided for at least 20 months [12]. If the patient fulfils the eligibility criteria for the standard shorter MDR-TB regimen (9-11 months) this can be used.

\section{EU-specific requirements}

As the treatment of MDR/XDR-TB often represents a last chance to ensure patient cure and survival, a full range of patient-centred measures, including counselling, observation and support of treatment, as well as psycho-social support are required to ensure adherence [12, 28-30]. This is particularly important given that these patients often belong to socially and economically disadvantaged groups.

For the treatment of MDR-TB, no drug should be administered to a patient with documented resistance (either by molecular or phenotypic DST). Thus, second-line DST should be performed to confirm the drug resistance pattern as well as to guide the correct choice of treatment.

In the EU/EEA, DST to ethambutol is considered reliable when conducted in quality-assured laboratories [76]. Pyrazinamide testing could be performed by genotypic (detection of pncA mutations) or phenotypic test (i.e. growth-based (liquid) automated methods).

The individualised regimen should include at least five effective TB medicines during the intensive phase, including pyrazinamide and four core second-line TB medicines. Drugs should be chosen as follows: one chosen from group $\mathrm{A}$, one from group $\mathrm{B}$, and at least two from group $\mathrm{C}$ (table 3 ). If the minimum number of five effective TB medicines cannot be composed from drugs included in groups A to $\mathrm{C}$, an agent from group D2 and other agents from group D3 may be added to bring the total to five. If pyrazinamide cannot be used (e.g. due to resistance or toxicity) an additional agent from group C or D can be added to strengthen the regimen. Total treatment duration ranges from 20 to 24 months, with the recommended intensive phase being 8 months [12]. 
TABLE 3 World Health Organization classification of anti-tuberculosis drugs [28]

\begin{tabular}{|c|c|c|}
\hline Group & Drugs & Abbreviation \\
\hline \multirow[t]{3}{*}{ A: Fluoroquinolones } & Levofloxacin & Lfx \\
\hline & Moxifloxacin & $M f x$ \\
\hline & Gatifloxacin & Gfx \\
\hline \multirow[t]{3}{*}{ B: Second-line injectables } & Amikacin & Am \\
\hline & Capreomycin & $\mathrm{Cm}$ \\
\hline & Kanamycin (streptomycin) & $\mathrm{Km}(\mathrm{S})$ \\
\hline \multirow[t]{4}{*}{ C: Other core second-line agents } & Ethionamide/prothionamide & Eto/Pto \\
\hline & Cycloserine/terizidone & $\mathrm{Cs} / \mathrm{Trd}$ \\
\hline & Linezolid & Lzd \\
\hline & Clofazimine & $\mathrm{Cfz}$ \\
\hline \multirow{9}{*}{$\begin{array}{l}\text { D: Add-on agents (not part of the core MDR-TB } \\
\text { regimen) }\end{array}$} & D1 Pyrazinamide & Z \\
\hline & Ethambutol & $\mathrm{E}$ \\
\hline & High-dose isoniazid & $\mathrm{H}_{\text {(high-dose) }}$ \\
\hline & D2 Bedaquiline & Bdq \\
\hline & Delamamid & Dlm \\
\hline & D3 $p$-aminosalicylic acid & PAS \\
\hline & Imipenem-cilastatin & Ipm \\
\hline & Meropenem & $\mathrm{Mpm}$ \\
\hline & $\begin{array}{l}\text { Amoxicillin-clavulanate } \\
\text { (thioacetazone) }\end{array}$ & Amx-Clv (T) \\
\hline
\end{tabular}

In patients with rifampicin-resistant $\mathrm{TB}$ or $\mathrm{MDR}-\mathrm{TB}$, who have not been previously treated with second-line drugs and in whom resistance to fluoroquinolones and second-line injectable agents has been excluded or is considered highly unlikely, a shorter MDR-TB regimen of 9-11 months recommended by WHO may be used instead of the conventional individualised regimen [12, 29, 79].

Treatment with new medicines including bedaquiline and delamanid along with repurposed medicines like linezolid and clofazamine and second-line medicines to which the M. tuberculosis strain is likely to be sensitive is required for patients suffering from XDR-TB or those patients suffering from strains resistant to fluoroquinolones or second-line injectables [77, 80]. For patients with serious adverse events to fluoroquinolones or second-line injectables new and repurposed medicines can also be considered.

Adverse events following prescription of second-line drugs should be managed according to international recommendations with the aim of limiting the probability of losing an effective drug due to such adverse events [10]

Adverse events, as well as the decision to start, modify or interrupt a second-line regimen should be managed by a team of experts (e.g. "TB Consilium" or similar body,) and not by individual physicians, in order to minimise mistakes and share responsibilities as well as share experience and expertise [77, 81, 82]. All efforts should be made to avoid development of further drug resistance.

WHO suggested that, in addition to chemotherapy, surgery may be used in selected cases with pulmonary TB, e.g. those with large cavities confined to one lobe [28]. Further research in this direction is necessary.

Notes

In order to prevent the selection of resistant $M$. tuberculosis mutants it is essential to never add only one effective drug to a failing regimen [28].

The "shorter regimen" recommended by WHO is as follows:

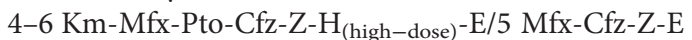

Note that in the original Bangladesh study gatifloxacin and not moxifloxacin was used [28].

The WHO criteria preventing the use of the "shorter regimen" include [12, 28, 29, 62, 76, 83-93]: 1) confirmed resistance or presumed ineffectiveness to a medicine in the shorter MDR-TB regimen; 2) exposure to $\geqslant 1$ second-line medicines in the shorter MDR-TB regimen for $>1$ month; 3 ) intolerance to $\geqslant 1$ medicines in the shorter MDR-TB regimen or risk of toxicity (e.g. drug-drug interactions); 4) pregnancy; 5) extrapulmonary disease; and 6) at least one medicine in the shorter MDR-TB regimen not available.

In Europe, the growth-based liquid DST (e.g. the mycobacteria growth indicator tube (MGIT) system) for ethambutol is considered reliable. Therefore, it might be considered an element of judgement for eligibility 
to the "shorter regimen" [75]. Line probe assays can be used as well, but there are mutations in additional genes conferring resistance to ethambutol that are not included in these assays. Ethionamide resistance can be predicted by molecular tests if mutations in inhA or EthA genes are detected by WGS. The association of inhA mutations with kat $G$ mutations makes the strains resistant to any dose of isoniazid [29, 79].

The American Thoracic Society/Infectious Diseases Society of America/Centers for Disease Control and Prevention TB treatment guidelines support the use of treatment drug monitoring in different situations, including difficult-to-treat MDR-TB cases $[26,27]$.

Given the difficulties in managing patients with rifampicin resistance or MDR-TB, and deciding correctly on the eligibility to the shorter regimen, referral of these patients to specialised centres is suggested.

\section{Standard 13 (changed)}

A written or electronic record of all medications administered, treatment monitoring (including bacteriologic response), adverse reactions and treatment outcomes, should be maintained for all patients.

\section{EU-specific requirements}

At the first contact with each patient, the complete clinical and social history on TB should be collected and included in the medical records. It should include the available information on previous diagnosis, treatment (regimen, doses, duration, changes in the regimen, etc.) and adherence, as well as complete information on bacteriology at diagnosis and during follow-up (sputum smear, culture and species identification, drug susceptibility testing for first and second-line drugs). This information should be reported in the documentation released to the patient (discharge letter, transfer-out form or equivalent document) to facilitate continuum of care if the patient moves/is moved to another health unit [94, 95].

Notes

Reporting forms for the described documentation can be obtained from several sources [23].

WHO has recommended countries to activate aDSM (active tuberculosis drug-safety monitoring and management) $[12,96,97]$.

Depending on resources availability, healthcare providers should consider to follow up MDR-TB cases after treatment completion to detect early relapse [98].

\section{Standards for addressing HIV infection and comorbidities Standard 14 (changed)}

HIV counselling should be carried out and HIV testing should be recommended to all patients with, or presumed to have, tuberculosis. Testing is of special importance as part of the routine management of all patients in areas with a high prevalence of HIV infection in the general population, or if the patient is from a high-risk population or has symptoms and/or signs of HIV-related conditions. Because of the close interaction between tuberculosis and HIV infection, integrated approaches to prevention and treatment of both infections are recommended [99].

\section{Standard 15 (changed)}

All patients with tuberculosis and HIV infection should be carefully evaluated: antiretroviral therapy is recommended in all HIV-positive tuberculosis patients. Tuberculosis treatment should be started immediately and the antiretroviral treatment prescribed as soon as possible.

\section{Notes}

The consideration of treatment with co-trimoxazole was retracted from the ESTC as they refer to HIV management and prevention of other infections. General prophylactic treatment against other infections is not relevant in the EU setting. Rather, the risk of HIV-related infections must be considered individually for each patient based on risk factors and setting, and form the basis for decision to provide prophylactic treatment against infections other than tuberculosis.

The 2017 WHO treatment guidelines suggest a delay between the initiation of TB therapy and the start of antiretroviral treatment of at least 14 days to reduce the risk of paradoxical reactions due to immune reconstitution syndrome $[12,30]$.

\section{Standard 16 (changed)}

Persons with HIV co-infection who, after careful evaluation, have a positive test (TST and/or IGRAs) for presumed latent infection with $M$. tuberculosis but do not have active tuberculosis should be offered preventive treatment. 


\section{EU specific requirements}

As HIV co-infection is known to increase the probability of developing active TB disease upon infection, HIV-seropositive persons who have been in contact with an index case harbouring an MDR-TB strain should initially undergo an individual risk assessment. Regular clinical monitoring and follow-up should be provided for those with evidence of latent infection [100].

Preventive treatment should take into account the drug resistance pattern of the source case, the CD4 count and the use of antiretroviral treatment. Preventive treatment should be provided with 6-month isoniazid, or 9-month isoniazid, or a 3-month regimen of weekly rifapentine plus isoniazid, or 3-4-month isoniazid plus rifampicin, or 3-4-month rifampicin alone [31, 101]. Rifampicin- and rifapentine-containing regimens should be prescribed with caution to people living with HIV who are on antiretroviral treatment due to potential drug-to-drug interactions [31, 32]

\section{Standard 17 (changed)}

All providers should conduct a thorough assessment of conditions that could affect tuberculosis treatment response or outcome. At the time the case management plan is developed, the provider should identify additional services that would support an optimal outcome for each patient and incorporate these services into an individualised plan of care. This plan should include assessment of and referrals for treatment for other illnesses with particular attention to those known to affect treatment outcome, for instance HIV, diabetes mellitus, drug and alcohol addiction, tobacco smoking, and other psycho-social problems [102]. Services such as antenatal or well-baby care should also be provided when needed.

\section{EU specific requirements}

Implementation of the entire package described in the WHO Interim Policy on Collaborative TB/HIV activities should be performed for all the activities, both those covered here and others included in the WHO package [99].

\section{Standards for public health and tuberculosis prevention Standard 18 (changed)}

All care providers for patients with tuberculosis should ensure that persons who have been in close contact with active and infectious $\mathrm{TB}$ patients are evaluated and managed in line with international recommendations. Close contacts include household and family members, and individuals with intensive or prolonged contact in congregate settings like prisons, homeless or migrant shelters, and indoor spaces like schools or offices

The risk of tuberculosis transmission depends on the concentration of the tubercle bacilli in the air, the airflow, the duration of the contact and the susceptibility of the contact to infection. The determination of priorities for contact investigation is based on the likelihood that a contact: 1) has undiagnosed and hence untreated tuberculosis; 2) is at high risk of having been infected by the index case; 3) is at high risk of developing tuberculosis if infected; and 4) is at risk of having severe tuberculosis if the disease develops.

\section{EU specific requirements}

The determinants of TB transmission and susceptibility should be carefully considered when assessing whether transmission has likely occurred and the need for initiating contact tracing [34].

Close contacts of MDR- and XDR-TB patients should be tested for LTBI and TB according to national guidelines. Contacts in which TB disease has been excluded and who are diagnosed with LTBI should undergo an individual risk assessment to determine: 1) the contact's risk for progression to TB disease; 2) the drug susceptibility pattern of the source case; and 3) the contact's risk for adverse events if initiating LTBI treatment $[31,32]$. Irrespective of the clinical advice regarding LTBI treatment, these contacts should be provided with careful clinical observation, information and health education by healthcare workers experienced in management of LTBI and TB disease [100, 103].

Involvement of local, community-based organisations (including community healthcare workers, non-clinical professionals and peers), is advisable when conducting contact tracing among vulnerable and hard-to-reach populations. This approach can contribute to the successful identification of potential contacts $[55,104]$

Clinicians and national programme managers are to interact with the relevant health authorities of host and/or home countries of TB patients belonging to migrant groups or mobile populations, to ensure continuum of care and contact investigation as appropriate [105]. 


\section{Standard 19 (changed)}

Contacts of an infectious tuberculosis patient, persons with HIV-infection, patients initiating anti-tumour necrosis factor (TNF) treatment, patients receiving dialysis, patients preparing for organ or haematological transplantation, and patients with silicosis should be tested for latent tuberculosis infection. If latent tuberculosis infection is identified they should be carefully evaluated for active tuberculosis. When active tuberculosis is excluded, preventive treatment using a WHO-recommended regimen should be offered.

\section{EU specific requirements}

Clinicians should collaborate with public health authorities in implementing adequate contact tracing procedures, performed according to national and international recommendations on progressive circles, when an infectious index case is diagnosed and notified [34, 103, 106, 107]. Similarly, both source finding and contact investigation should be initiated if a child with TB (any site of infection) has been identified and where no source has been identified $[103,108]$.

Individuals undergoing treatment with anti-TNF- $\alpha$ should be considered as high-risk contacts. According to the ESTC number 16, in individuals who are HIV-infected or affected by comorbidities, treatment of latent infection should be promptly initiated if TB infection is identified by TST and/or IGRAs and active tuberculosis disease is excluded [31, 32, 34, 63, 109, 110].

\section{Notes}

As indicated in ESTC number 18, a comprehensive individual risk assessment and close clinical monitoring should be provided to close contacts of an MDR-TB or XDR-TB source case irrespective of the clinical advice regarding LTBI treatment $[31,100]$.

Treatment for LTBI should be according to national and international recommendations, as outlined in ESTC number $16[31,107]$

\section{Standard 20 (changed)}

Each healthcare facility caring for patients who have, or are presumed to have infectious tuberculosis, should develop and implement an appropriate tuberculosis infection control plan.

\section{EU specific requirements}

Community-based treatment, supported by infection control measures at home, should be available for patients preferring to undergo treatment at home $[12,30]$.

If hospitalisation is required, clinicians should ensure that all newly admitted patients who are presumed to have infectious TB are subject to respiratory isolation until their diagnosis is confirmed or excluded [10].

In order to prevent transmission of tubercle bacilli to other patients, staff and/or visitors, smear-positive TB patients should ideally be isolated in appropriate rooms until they achieve bacteriological conversion (negative sputum microscopy). Isolation should be in rooms with negative pressure ventilation.

An appropriate infection control plan, managed by a designated person, should include the following four components; managerial activities; administrative controls; environmental controls; and personal protection interventions $[12,111]$. Adequate administrative measures for tuberculosis infection control should be in place in all healthcare facilities, as well as adequate respiratory protection measures (including the use of respirators following respirator fit testing for staff and the use of surgical mask for infectious patients). Appropriate training on infection control to staff, and standardised health education of patients on cough etiquette, based on validated tools, should be also included in the infection control plan. Infection control committees, which cover airborne diseases, and includes infection control experts, should also be implemented $[12,111-113]$.

\section{Notes}

The implementation of an infection control plan is essential for the treating clinician and health facility as well as for the overall health system. Clinicians should maintain a dialogue within their health facility, develop a sound infection control plan, contributing with their technical expertise. The health facility should engage with all healthcare workers, non-medical staff, patients and visitors and ensure optimal implementation, practice and monitoring of these infection control measures; all healthcare workers should be (re)trained in the infection control plan [10, 111, 114-116].

It is important to have a designated infection control focal person with the required authority to ensure the implementation of the infection control plan. 
With regard to the need of isolating infectious tuberculosis patients, it is important to consider several options for isolation, and not only that of hospitalisation. For example, a patient with drug-susceptible TB who can be treated at home (i.e. no need for hospitalisation due to severe health status), does not need to be hospitalised, as long as appropriate measures for treatment and infection control are ensured at the residence $[111,117,118]$.

Patients with a clinical indication for hospital admission, such as comorbidities, should not be hospitalised in a general medical ward. Such patients should be placed in rooms that allow appropriate respiratory isolation.

\section{Standard 21 (unchanged)}

All providers must report both new and re-treatment tuberculosis cases and their treatment outcomes to local public health authorities, in conformance with applicable legal requirements and policies.

\section{EU specific requirements}

Clinicians should perform treatment outcome evaluations in their clinical unit at regular time intervals (e.g. quarterly) $[23,119]$. Treatment outcomes should be reported to local public health authorities, in conformance with applicable requirements and policies and, at the same time, be used as a monitoring and evaluation tool to improve the quality of patient management. Information on treatment outcome should also regularly be channelled back from the public health department to the healthcare providers, to allow a coordinated evaluation of the outcomes. Information on the final outcome of patients should be available at the clinical unit which initiated treatment, even when the patient is transferred out. Adequate training must be provided to health staff in charge of reporting treatment outcomes to public health authorities and performing the quarterly evaluation of the clinic's own cases. This principle is also applicable to TB patients moving across EU borders [1, 120-122].

\section{Supporting enablers to the ESTC}

In the process of developing the standards and reaching consensus, the panel of experts identified the need and added-value of identifying and listing "supporting enablers" to the standards. These are a resource for policymakers, clinicians, public health workers and other stakeholders to identify how best to adopt, adapt, introduce and implement the ESTCs in their setting with the ultimate goal of securing optimal TB care, prevention and control

1) Formal adoption of the European Union Standards for Tuberculosis Care, for the care, prevention and control at national level. This current version of the ESTC is translated into all EU languages, thus facilitating endorsement by national medical associations and incorporation of the ESTC into training curricula of health staff [123].

2) Development of consistent tuberculosis control and elimination strategies and policies according to the principles described in the End TB Strategy $[2,20,22]$ and Tuberculosis Action Plan for WHO European Region 2016-2020 [21], taking into account best practices in and outside Europe [124, 125].

3) Adoption of specific, updated, evidence-based tuberculosis and multidrug-resistant TB guidelines, together with mechanisms to update them on a regular basis and to monitor their implementation (auditand or knowledge, attitudes and practices study (KAP study)-based) [22, 126-128].

4) Planning and organisation of an adequate national laboratory network to ensure that a minimum sufficient number of mycobacteriology laboratories are in place, allowing implementation of the standards described in this document (adequate coverage of the country, adequate internal and external quality assurance procedures in place, sufficient numbers of samples per laboratory to ensure proficiency, availability of national laboratories with reference functions to support regional and local laboratories, etc.) $[17,18,24]$.

5) Development of policies ensuring the registration and continuous availability of all first- and second-line TB drugs and add-on agents (e.g. through coordinated procurement with partner countries for the drugs not registered in the country or which are necessary in small quantities) [22].

6) Securing consistent and adequate funding for TB and MDR/XDR-TB care, prevention and control that is sufficient to run the activities mentioned in this document. This should include psycho-social support and coordination of care for all patients, as highlighted in the International Patients' Charter for rights to diagnosis and treatment. This applies particularly to patients belonging to vulnerable populations $[22,69,129]$.

Acknowledgements: The authors wish to thank the ERS Office, Lausanne, Switzerland, for overall assistance making this effort possible; Iuliana Dobre, Simona Baban and Marius Dumitru from the Romanian Association of TB Patients (ARB 
TB) for their comments on the manuscript; and Blagovesta Gavazova and Donka Stefanova (Bulgaria) for their contribution in the ERS/ECDC survey performed in their country.

Conflict of interest: S. Aliberti reports grants and personal fees from Bayer Healthcare, Aradigm Corporation, Grifols, Chiesi and INSMED, and personal fees from AstraZeneca, Basilea, Zambon, Novartis, Raptor, Actavis UK Ltd and Horizon, outside the submitted work.

Support statement: The development of the ESTC was financially supported by the European Respiratory Society (ERS) through core funds via the guidelines development scheme and by the European Centre for Disease Prevention and Control (ECDC). The ERS development group (G. Sotgiu, G.B. Migliori, R. Centis, L. D'Ambrosio and R. Duarte) and the ECDC (M.J. van der Werf and S. Rosales-Klintz) wrote the first draft. All authors then reviewed the draft document and contributed revisions and supporting references. Funding information for this article has been deposited with the Crossref Funder Registry.

\section{References}

1 European Centre for Disease Prevention and Control/WHO Regional Office for Europe. Tuberculosis surveillance and monitoring in Europe 2018-2016 data. Stockholm, European Centre for Disease Prevention and Control, 2018. Available from: https://ecdc.europa.eu/sites/portal/files/documents/ecdc-tuberculosis-surveillance-monitoringEurope-2018-19mar2018.pdf

2 Lonnroth K, Migliori GB, Abubakar I, et al. Towards tuberculosis elimination: an action framework for low-incidence countries. Eur Respir J 2015; 45: 928-952.

3 D'Ambrosio L, Dara M, Tadolini M, et al. Tuberculosis elimination: theory and practice in Europe. Eur Respir J 2014; 43: 1410-1420.

4 van der Werf MJ, Kodmon C, Hollo V, et al. Drug resistance among tuberculosis cases in the European Union and European Economic Area, 2007 to 2012. Euro Surveill 2014; 19: 20733.

5 van der Werf MJ, Kodmon C, Zucs P, et al. Tuberculosis and HIV coinfection in Europe: looking at one reality from two angles. AIDS 2016; 30: 2845-2853.

6 van der Werf MJ, Sotgiu G, Dara M. Closing the gap in surveillance of tuberculosis and HIV co-infection: a European perspective on the need for clinician-public health alliances. Eur Respir J 2017; 50: 1701758.

7 van der Werf MJ, Erkens C, Gebhard A, et al. First joint meeting of three European tuberculosis networks. Euro Surveill 2013; 18: 20583.

8 Migliori GB, Zellweger JP, Abubakar I, et al. European union standards for tuberculosis care. Eur Respir J 2012; 39: $807-819$.

9 Tuberculosis Coalition for Technical Assistance. International Standards for Tuberculosis Care (ISTC). 2nd edn The Hague, Tuberculosis Coalition for Technical Assistance, 2009.

10 TB CARE I. International Standards for Tuberculosis Care. 3rd edn. The Hague, TB CARE, 2014.

11 Migliori GB, Sotgiu G, Blasi F, et al. Towards the development of EU/EEA Standards for Tuberculosis Care (ESTC). Eur Respir J 2011; 38: 493-495.

12 World Health Organization. Compendium of WHO guidelines and associated standards: ensuring optimum delivery of the cascade of care for patients with tuberculosis. Geneva, World Health Organization, 2017.

13 van der Werf MJ, Sandgren A, D’Ambrosio L, et al. The European Union standards for tuberculosis care: do they need an update? Eur Respir J 2014; 43: 933-942.

14 Sotgiu G, Beer N, Aliberti S, et al. Fighting tuberculosis in the EU/EEA: towards the new European Union standards on tuberculosis care. Eur Respir J 2016; 48: 1278-1281.

15 Migliori GB, Sotgiu G, D'Ambrosio L, et al. TB and MDR/XDR-TB in European Union and European Economic Area countries: managed or mismanaged? Eur Respir J 2012; 39: 619-625.

16 Drobniewski FA, Hoffner S, Rusch-Gerdes S, et al. Recommended standards for modern tuberculosis laboratory services in Europe. Eur Respir J 2006; 28: 903-909.

17 World Health Organization Regional Office for Europe. Algorithm for laboratory diagnosis and treatment-monitoring of pulmonary tuberculosis and drug-resistant tuberculosis using state-of-the-art rapid molecular diagnostic technologies. Expert opinion of the European Tuberculosis Laboratory Initiative core group members for the WHO European Region. Copenhagen, WHO Regional Office for Europe, 2017.

18 European Centre for Disease Prevention and Control. Handbook on TB laboratory diagnostic methods for the European Union. Stockholm, ECDC, 2016.

19 Veen J, Migliori GB, Raviglione M, et al. Harmonisation of TB control in the WHO European region: the history of the Wolfheze Workshops. Eur Respir J 2011; 37: 950-959.

20 World Health Organization, European Respiratory Society. Framework towards tuberculosis elimination in low-incidence countries. Geneva, World Health Organization, 2014.

21 World Health Organization Regional Office for Europe. Roadmap to implement the tuberculosis action plan for the European Region 2016-2020. Towards ending tuberculosis and multidrug-resistant tuberculosis. Copenhagen, WHO Regional Office for Europe, 2016.

22 World Health Organization. Implementing the end TB strategy: the essentials. Geneva, World Health Organization, 2015.

23 World Health Organization. Definitions and reporting framework for tuberculosis - 2013 revision. Geneva, Word Health Organization, 2013.

24 World Health Organization. Implementing tuberculosis diagnostics. Policy framework. Geneva, World Health Organization, 2015.

25 Lewinsohn DM, Leonard MK, LoBue PA, et al. Official American Thoracic Society/Infectious Diseases Society of America/Centers for Disease Control and Prevention Clinical Practice Guidelines: Diagnosis of Tuberculosis in Adults and Children. Clin Infect Dis 2017; 64: e1-e33.

26 Nahid P, Dorman SE, Alipanah N, et al. Official American Thoracic Society/Centers for Disease Control and Prevention/Infectious Diseases Society of America Clinical Practice Guidelines: treatment of drug-susceptible tuberculosis. Clin Infect Dis 2016; 63: e147-e195. 
Sotgiu G, Nahid P, Loddenkemper R, et al. The ERS-endorsed official ATS/CDC/IDSA clinical practice guidelines on treatment of drug-susceptible tuberculosis. Eur Respir J 2016; 48: 963-971.

World Health Organization. WHO treatment guidelines for drug-resistant tuberculosis: 2016 update. Geneva World Health Organization, 2016.

Falzon D, Schunemann HJ, Harausz E, et al. World Health Organization treatment guidelines for drug-resistant tuberculosis, 2016 update. Eur Respir J 2017; 49: 1602308. World Health Organization. Guidelines on the management of latent tuberculosis infection. 2015.

32 Getahun H, Matteelli A, Abubakar I, et al. Management of latent Mycobacterium tuberculosis infection: WHO guidelines for low tuberculosis burden countries. Eur Respir J 2015; 46: 1563-1576.

33 Storla DG, Yimer S, Bjune GA. A systematic review of delay in the diagnosis and treatment of tuberculosis. BMC Public Health 2008; 8: 15.

34 Erkens CG, Kamphorst M, Abubakar I, et al. Tuberculosis contact investigation in low prevalence countries: a European consensus. Eur Respir J 2010; 36: 925-949.

35 European Respiratory Society, European Lung Foundation. Lung Health in Europe Facts and Figures. Loddenkemper R, Gibson G, Sibille Y, eds. Sheffield, European Respiratory Society, 2003.

36 Schwoebel V, Lambregts CS, Moro ML, et al. European recommendations on surveillance of antituberculosis drug resistance. Euro Surveill 2000; 5: 104-106.

37 World Health Organization. Multidrug and extensively drug-resistant TB (M/XDR-TB) : 2010 global report on surveillance and response. Geneva, World Health Organization, 2010.

38 Pai M, Behr MA, Dowdy D, et al. Tuberculosis. Nat Rev Dis Primers 2016; 2: 16076

39 Walker TM, Kohl TA, Omar SV, et al. Whole-genome sequencing for prediction of Mycobacterium tuberculosis drug susceptibility and resistance: a retrospective cohort study. Lancet Infect Dis 2015; 15: 1193-1202.

40 Kendall EA, Cohen T, Mitnick CD, et al. Second line drug susceptibility testing to inform the treatment of rifampin-resistant tuberculosis: a quantitative perspective. Int J Infect Dis 2017; 56: 185-189.

41 Trajman A, Durovni B, Saraceni V, et al. High positive predictive value of Xpert in a low rifampicin resistance prevalence setting. Eur Respir J 2014; 44: 1711-1713.

42 Albert H, Nathavitharana RR, Isaacs C, et al. Development, roll-out and impact of Xpert MTB/RIF for tuberculosis: what lessons have we learnt and how can we do better? Eur Respir J 2016; 48: 516-525.

43 Chakravorty S, Simmons AM, Rowneki M, et al. The New Xpert MTB/RIF Ultra: improving detection of Mycobacterium tuberculosis and resistance to rifampin in an assay suitable for point-of-care testing. $m B i o$ 2017; 8: e00812-17.

44 Nathavitharana RR, Cudahy PG, Schumacher SG, et al. Accuracy of line probe assays for the diagnosis of pulmonary and multidrug-resistant tuberculosis: a systematic review and meta-analysis. Eur Respir J 2017; 49: 1601075.

45 Theron G, Peter J, Richardson M, et al. GenoType(R) MTBDRsl assay for resistance to second-line anti-tuberculosis drugs. Cochrane Database Syst Rev 2016; 9: CD010705.

46 Sehgal IS, Dhooria S, Aggarwal AN, et al. Diagnostic performance of Xpert MTB/RIF in tuberculous pleural effusion: systematic review and meta-analysis. J Clin Microbiol 2016; 54: 1133-1136.

47 Ling DI, Zwerling AA, Pai M. GenoType MTBDR assays for the diagnosis of multidrug-resistant tuberculosis: a meta-analysis. Eur Respir J 2008; 32: 1165-1174.

48 Pai M, Nicol MP, Boehme CC. Tuberculosis diagnostics: state of the art and future directions. Microbiol Spectr 2016; 4: TBTB2-0019-2016.

49 Boehme CC, Nabeta P, Hillemann D, et al. Rapid molecular detection of tuberculosis and rifampin resistance. N Engl J Med 2010; 363: 1005-1015.

50 Boehme CC, Nicol MP, Nabeta P, et al. Feasibility, diagnostic accuracy, and effectiveness of decentralised use of the Xpert MTB/RIF test for diagnosis of tuberculosis and multidrug resistance: a multicentre implementation study. Lancet 2011; 377: 1495-1505.

51 Pankhurst LJ, Del Ojo Elias C, Votintseva AA, et al. Rapid, comprehensive, and affordable mycobacteria diagnosis with whole-genome sequencing: a prospective study. Lancet Respir Med 2016; 4: 49-58.

52 Walker TM, Cruz ALG, Peto TE, et al. Tuberculosis is changing. Lancet Infect Dis 2017; 17: 359-361.

53 Miotto P, Tessema B, Tagliani E, et al. A standardised method for interpreting the association between mutations and phenotypic drug-resistance in Mycobacterium tuberculosis. Eur Respir J 2017; 50: 1701354.

54 Falzon D, Jaramillo E, Schunemann HJ, et al. WHO guidelines for the programmatic management of drug-resistant tuberculosis: 2011 update. Eur Respir J 2011; 38: 516-528.

55 National Instititute for Health and Care Excellence. Tuberculosis (NG33). London, NICE, 2016.

56 World Health Organization. Toman's tuberculosis. Case detection, treatment, and monitoring- questions and answers. Frieden T, ed. Geneva, World Health Organization, 2004.

57 Datta S, Shah L, Gilman RH, et al. Comparison of sputum collection methods for tuberculosis diagnosis: a systematic review and pairwise and network meta-analysis. Lancet Glob Health 2017; 5: e760-e771.

58 World Health Organization. Rapid Advice: Treatment of Tuberculosis in Children. Geneva, World Health Organization, 2010.

59 Seddon JA, Furin JJ, Gale M, et al. Caring for children with drug-resistant tuberculosis: practice-based recommendations. Am J Respir Crit Care Med 2012; 186: 953-964.

60 Schoch OD, Rieder P, Tueller C, et al. Diagnostic yield of sputum, induced sputum, and bronchoscopy after radiologic tuberculosis screening. Am J Respir Crit Care Med 2007; 175: 80-86.

61 European Centre for Disease Prevention and Control. Use of interferon-gamma release assays in support of TB diagnosis. Stockholm, ECDC, 2011.

62 Sotgiu G, Tiberi S, D'Ambrosio L, et al. WHO recommendations on shorter treatment of multidrug-resistant tuberculosis. Lancet 2016; 387: 2486-2487.

63 Diel R, Goletti D, Ferrara G, et al. Interferon-gamma release assays for the diagnosis of latent Mycobacterium tuberculosis infection: a systematic review and meta-analysis. Eur Respir J 2011; 37: 88-99. 
Sester M, Sotgiu G, Lange C, et al. Interferon-gamma release assays for the diagnosis of active tuberculosis: a systematic review and meta-analysis. Eur Respir J 2011; 37: 100-111.

Petruccioli E, Scriba TJ, Petrone L, et al. Correlates of tuberculosis risk: predictive biomarkers for progression to active tuberculosis. Eur Respir J 2016; 48: 1751-1763.

Goletti D, Petruccioli E, Joosten SA, et al. Tuberculosis biomarkers: from diagnosis to protection. Infect Dis Rep 2016; 8: 6568.

World Health Organization. Guidance for national tuberculosis programmes on the management of tuberculosis in children. 2nd edn. Geneva, World Health Organization, 2014.

World Health Organization. Report of a WHO consultation on strengthening the active engagement of civil society organizations in the global TB prevention, care and control efforts. Geneva, World Health Organization, 2010. Report No.: WHO/HTM/TB/2010.15.

World Care Council. Patients' Charter for Tuberculosis Care. Viols en Laval, World Care Council, 2006.

Munro SA, Lewin SA, Smith HJ, et al. Patient adherence to tuberculosis treatment: a systematic review of qualitative research. PLoS Med 2007; 4: e238.

Volmink J, Matchaba P, Garner P. Directly observed therapy and treatment adherence. Lancet 2000; 355: 1345-1350.

Mitnick CD, White RA, Lu C, et al. Multidrug-resistant tuberculosis treatment failure detection depends on monitoring interval and microbiological method. Eur Respir J 2016; 48: 1160-1170.

Lange C, Duarte R, Frechet-Jachym M, et al. Limited benefit of the new shorter multidrug-resistant tuberculosis regimen in europe. Am J Respir Crit Care Med 2016; 194: 1029-1031.

Sotgiu G, Tiberi S, D'Ambrosio L, et al. Faster for less, the new "Shorter" regimen for multidrug-resistant tuberculosis. Eur Respir J 2016; 48: 1503-1507.

van der Werf MJ, Hollo V, Kodmon C, et al. Eligibility for shorter treatment of multidrug-resistant tuberculosis in the European Union. Eur Respir J 2017; 49: 1601992.

van der Werf MJ, Kodmon C, Catchpole M, et al. Shorter regimens for multidrug-resistant tuberculosis should also be applicable in Europe. Eur Respir J 2017; 49: 1700463.

Esposito S, D'Ambrosio L, Tadolini M, et al. ERS/WHO Tuberculosis Consilium assistance with extensively drug-resistant tuberculosis management in a child: case study of compassionate delamanid use. Eur Respir $J$ 2014; 44: 811-815.

van Altena R, Akkerman OW, Alffenaar JC, et al. Shorter treatment for multidrug-resistant tuberculosis: the good, the bad and the ugly. Eur Respir J 2016; 48: 1800-1802.

Caminero JA, Piubello A, Scardigli A, et al. Proposal for a standardised treatment regimen to manage pre- and extensively drug-resistant tuberculosis cases. Eur Respir J 2017; 50: 1700648.

Borisov SE, Dheda K, Enwerem M, et al. Effectiveness and safety of bedaquiline-containing regimens in the treatment of MDR- and XDR-TB: a multicentre study. Eur Respir J 2017; 49: 1700387.

Blasi F, Dara M, van der Werf MJ, et al. Supporting TB clinicians managing difficult cases: the ERS/WHO Consilium. Eur Respir J 2013; 41: 491-494.

Tadolini M, Garcia-Prats AJ, D'Ambrosio L, et al. Compassionate use of new drugs in children and adolescents with multidrug-resistant and extensively drug-resistant tuberculosis: early experiences and challenges. Eur Respir J 2016; 48: 938-943.

Aung KJ, Van Deun A, Declercq E, et al. Successful "9-month Bangladesh regimen" for multidrug-resistant tuberculosis among over 500 consecutive patients. Int J Tuberc Lung Dis 2014; 18: 1180-1187.

Kuaban C, Noeske J, Rieder HL, et al. High effectiveness of a 12-month regimen for MDR-TB patients in Cameroon. Int J Tuberc Lung Dis 2015; 19: 517-524.

Piubello A, Harouna SH, Souleymane $\mathrm{MB}$, et al. High cure rate with standardised short-course multidrug-resistant tuberculosis treatment in Niger: no relapses. Int J Tuberc Lung Dis 2014; 18: 1188-1194.

Van Deun A, Maug AK, Salim MA, et al. Short, highly effective, and inexpensive standardized treatment of multidrug-resistant tuberculosis. Am J Respir Crit Care Med 2010; 182: 684-692.

Heldal E, van Deun A, Chiang CY, et al. Shorter regimens for multidrug-resistant tuberculosis should also be applicable in Europe. Eur Respir J 2017; 49: 1700228.

Javaid A, Ahmad N, Khan AH, et al. Applicability of the World Health Organization recommended new shorter regimen in a multidrug-resistant tuberculosis high burden country. Eur Respir J 2017; 49: 1601967.

Van Deun A, Chiang CY. Shortened multidrug-resistant tuberculosis regimens overcome low-level fluoroquinolone resistance. Eur Respir J 2017; 49: 1700223.

Dalcolmo M, Gayoso R, Sotgiu G, et al. Resistance profile of drugs composing the "shorter" regimen for multidrug-resistant tuberculosis in Brazil, 2000-2015. Eur Respir J 2017; 49: 1602309.

Chee CBE, KhinMar KW, Sng L, et al. The shorter multidrug-resistant tuberculosis treatment regimen in Singapore: are patients from South-East Asia eligible? Eur Respir J 2017; 50: 1700753.

Kendall EA, Fojo AT, Dowdy DW. Expected effects of adopting a 9 month regimen for multidrug-resistant tuberculosis: a population modelling analysis. Lancet Respir Med 2017; 5: 191-199.

Sotgiu G, Migliori G. Effect of the short-course regimen on the global epidemic of multidrug-resistant tuberculosis. Lancet Respir Med 2017; 5: 159-161.

Lange C, Abubakar I, Alffenaar JW, et al. Management of patients with multidrug-resistant/extensively drug-resistant tuberculosis in Europe: a TBNET consensus statement. Eur Respir J 2014; 44: 23-63.

Caminero JA. Management of multidrug-resistant tuberculosis and patients in retreatment. Eur Respir J 2005; 25: 928-936.

World Health Organization. Active tuberculosis drug-safety monitoring and management (aDSM). Framework for implementation. Geneva, World Health Organization, 2015.

Halleux CM, Falzon D, Merle C, et al. The WHO global aDSM database: generating evidence on the safety of new treatment regimens for drug-resistant tuberculosis. Eur Respir J 2018; 51: 1701643.

Gunther G, Lange C, Alexandru S, et al. Treatment outcomes in multidrug-resistant tuberculosis. N Engl J Med 2016; 375: 1103-1105.

World Health Organization. WHO policy on collaborative TB/HIV activities: guidelines for national programmes and other stakeholders. Geneva, World Health Organization, 2012. 

patients. Stockholm, ECDC, 2012.

101 Zenner D, Beer N, Harris RJ, et al. Treatment of latent tuberculosis infection: an updated network meta-analysis. Ann Intern Med 2017; 167: 248-255.

102 Creswell J, Raviglione M, Ottmani S, et al. Tuberculosis and noncommunicable diseases: neglected links and missed opportunities. Eur Respir J 2011; 37: 1269-1282.

103 Luzzati R, Migliori G, Zignol M, et al. Children under 5 years are at risk for tuberculosis after occasional contact with highly contagious patients: an out-patient outbreak from a smear-positive healthcare worker. Eur Respir $J$ 2017; 50: 1701414 .

104 European Centre for Disease Prevention and Control. Guidance on tuberculosis control in vulnerable and hard-to-reach populations. Stockholm: ECDC, 2016.

105 Dara M, de Colombani P, Petrova-Benedict R, et al. Minimum package for cross-border TB control and care in the WHO European region: a Wolfheze consensus statement. Eur Respir J 2012; 40: 1081-1090.

106 Leung CC, Rieder HL, Lange C, et al. Treatment of latent infection with Mycobacterium tuberculosis: update 2010. Eur Respir J 2011; 37: 690-711.

107 Woldehanna S, Volmink J. Treatment of latent tuberculosis infection in HIV infected persons. Cochrane Database Syst Rev 2004; CD000171.

108 European Centre for Disease Prevention and Control. Investigation and control of tuberculosis incidents affecting children in congregate settings. Stockholm, ECDC, 2013.

109 Mack U, Migliori GB, Sester M, et al. LTBI: latent tuberculosis infection or lasting immune responses to M. tuberculosis? A TBNET consensus statement. Eur Respir J 2009; 33: 956-973.

110 Lange C, Rieder HL. Intention to test is intention to treat. Am J Respir Crit Care Med 2011; 183: 3-4.

111 World Health Organization. WHO policy on TB infection control in health - care facilities, congregate settings and households. Geneva, World Health Organization, 2009.

112 Sotgiu G, D’Ambrosio L, Centis R, et al. TB and M/XDR-TB infection control in European TB reference centres: the Achilles' heel? Eur Respir J 2011; 38: 1221-1223.

113 Matteelli A, Centis R, D’Ambrosio L, et al. WHO strategies for the programmatic management of drug-resistant tuberculosis. Expert Rev Respir Med 2016; 10: 991-1002.

114 Turusbekova N, Ljungqvist I, Davidaviciene E, et al. Tuberculosis infection control in health facilities in Lithuania: lessons learnt from a capacity support project. Public Health Action 2016; 6: 22-24.

115 Turusbekova N, Popa C, Dragos M, et al. Strengthening TB infection control in specialized health facilities in Romania--using a participatory approach. Public Health 2016; 131: 75-81.

116 Schmidt BM. Tuberculosis interventions to prevent transmission of infection in health care workers: a systematic review. Thesis, Cape Town, University of Cape Town, 2014.

117 Jensen PA, Lambert LA, Iademarco MF, et al. Guidelines for preventing the transmission of Mycobacterium tuberculosis in health-care settings, 2005. MMWR Recomm Rep 2005; 54: 1-141.

118 Acuña-Villaorduña C, Jones-Lopez E, Fregona G, et al. Intensity of exposure to pulmonary tuberculosis determines risk of tuberculosis infection and disease. Eur Respir J 2018; 51: 1701578.

119 Laserson KF, Thorpe LE, Leimane V, et al. Speaking the same language: treatment outcome definitions for multidrug-resistant tuberculosis. Int J Tuberc Lung Dis 2005; 9: 640-645.

120 Mor Z, Migliori GB, Althomsons SP, et al. Comparison of tuberculosis surveillance systems in low-incidence industrialised countries. Eur Respir J 2008; 32: 1616-1624.

121 Uplekar M, Atre S, Wells WA, et al. Mandatory tuberculosis case notification in high tuberculosis-incidence countries: policy and practices. Eur Respir J 2016; 48: 1571-1581.

122 Heldal E, Kuyvenhoven JV, Wares F, et al. Diagnosis and treatment of tuberculosis in undocumented migrants in low- or intermediate-incidence countries. Int J Tuberc Lung Dis 2008; 12: 878-888.

123 Williams G, Alarcon E, Jittimanee S, et al. Guidance for the implementation of best practice for the care of patients with tuberculosis. Int J Tuberc Lung Dis 2008; 12: 236-240.

124 Voniatis C, Migliori GB, Voniatis M, et al. Tuberculosis elimination: dream or reality? The case of Cyprus. Eur Respir J 2014; 44: 543-546.

125 Al Yaquobi F, Al-Abri S, Al-Abri B, et al. Tuberculosis elimination: a dream or reality? The case of Oman. Eur Respir J 2018; 51: 1702027 .

126 World Health Organization. Framework for the engagement of all health care providers in the management of drug resistant tuberculosis. Geneva, World Health Organization, 2015.

127 World Health Organization. Public-private mix for drug-resistant tuberculosis management: a situation assessment tool to engage all relevant care providers in drug-resistant tuberculosis (DR-TB) management at country level. Geneva, World Health Organization, 2015.

128 World Health Organization. Public-Private Mix for TB care and control. A tool for national situation assessment. Geneva, World Health Organization, 2007.

129 World Health Organization. Ethics guidance for the implementation of the End TB strategy. Geneva, World Health Organization, 2017. 